\title{
Recent Trends and Improvisations in FPGA
}

\author{
M. Joy Daniel, K. Siva Kumar M.E. \\ B.E. Electrical and Electronics Engineering, K. P. R. Institute of Engineering and Technology, India. \\ Assistant Professor, Department of Electrical and Electronics Engineering, \\ K. P. R. Institute of Engineering and Technology, India.
}

\begin{abstract}
With the improvements of VLSI design in FPGA, the application areas got wider. The FPGAs architectural developments afford FPGA developers to produce effective devices day-by-day. Today these applications wider from commercial-applications to cloud server, artificial intelligence, defense, medical, etc. In this paper, the various and recent trends and improvements of FPGA are produced. By System-on-chip development by using FPGA made easy the advancements in optical interconnects, hardware accelerators and controllers. The developers promote FPGA in photonics network and new architectures to provide speed and user defined specifications. FPGA and ASIC plays an important role in the advancements in IoT. The size of FPGAs is evolutionally breaking down by fuelling reprogrammable from $45 \mathrm{~nm}$ to $8 \mathrm{~nm}$. This paper also revealed some features in cloud computing using wavelength division multiplexing (WDM), optical network and silicon photonics.
\end{abstract}

Keywords: Field-programmable gate array (FPGA), Application Specific Integrated Circuits (ASIC), cloud servers, System-on-chip (SoC), wavelength division multiplexing (WDM) and Internet of Things (IoT).

\section{Introduction}

FPGAs are desirable over a typical CPU because they are faster and use less energy than a CPU. Input and output bandwidth, memory bandwidth and computation are the three performance vectors exceed the offering of conventional processors using performance of FPGA as a computing platform. Also they are customizable or reprogrammable. FPGA represents a programmable digital processor with numerous gates that enable system designs. Security in information and advanced FPGA programming tools are present fieldprogrammable gate array technology for aerospace and defense. These developing custom-programs provide the device on security, functionality and other benefits. The two different kinds of security is done by cryptography keep the information from decoding and anti-tamper. While the performance of FPGA increases, the complexity also increases in architecture ${ }^{[1]}$. FPGA Fabric, Block RAM, Embedded Registers and Multipliers, Clock management, Multi-standard programmable IO, Embedded microprocessor, Multi-gigabit transceivers, Embedded DSP-optimized multipliers and Embedded Ethernet MACs provided from Domain-optimized system logic. The cutting-edge trend in FPGA is the insertion of special hardware by the form of hard cores. Hard cores having dedicated physical components with high frequency and fixed implementation ${ }^{[13]}$. The resultant high-end Multiprocessors systems having high speed I/O, improved system for microprocessor development and FMC modules by adoption of FPGA. Other improvements such as scalability, re-configurability or affordability have been broaden applications, developing and custom devices for designers and entrepreneurs. Presently embedded processors are available in Hybrid FPGA/SOC are ready to be used in signal processing applications like video analysis algorithms, image processing etc. Standard processor system is combined with reconfigurable hardware for specific modules. The parallelization of hardware systems co-designed with pieces of software executed on one or more standard processors (Figure 1) ${ }^{[2]}$.

\section{II.1. FPGA As Cloud Server:}

\section{Recent trends in fpga}

IoT devices whose processing power, memory size and bandwidth are usually limited. The developers offering interfaces through compilers, tools, and frameworks. This creates effectiveness for the customer base and creates such as strong cloud products with increased reach. All kinds of new machine learning, AI, big data analysis is possible in one platform. Web Service Companies working to offer FPGAs in Elastic Compute Cloud (EC2) cloud environment. Government drive certain FPGA accelerated analytics business for healthcare and financial services. This analytics increased push over the gene sequencing anomalies ${ }^{[9]}$ (Figure 2). Designed FPGA-boosted analytics on the developed FPGA instances to booster elastic search will allow for such multiple datasets. These developed FPGAs has 2.4 million logic elements, which has a strong broad CPU and 6500 DSP engines. Strong host CPU by FPGAs is a much greater opportunities with partial reconfiguration with different and better analytical workloads ${ }^{[10]}$. For match the reconfiguration capabilities of the FPGAs with 
sources instead of streaming data from an SSD, get directly from S3 or Glacier or some other data source. Ring topology up to $400 \mathrm{~GB} / \mathrm{sec}$ and commercial workloads from very new FPGAs (Figure 3$)^{[11]}$.

\section{II.2.Artificial Intelligence:}

Artificial Intelligence demands for higher performance, less time, larger computation with more power proficient for deep neural networks ${ }^{[6]}$. Deep neural network power-up the high-end devices. As an order of higher magnitude performance per Watt than commercial FPGAs and (Graphical Processing Unit) GPUs in SOC search giant offers TPUs (Google's Tensor Processing Units). Google revealed that the accelerators were used for the Alpha GO systems which is a computer developed by Google DeepMind that plays the board game Go (Figure 4). Also CEA is offers an ultra-low power programmable accelerator called P-Neuro. P-Neuro is built on clustered SIMD architecture (highly configure neural architecture) with optimized memory hierarchy and interconnections. P-Neuro based on FPGA running at $100 \mathrm{MHz}$ faster by a factor of two, four to five times more energy efficient compared to the embedded GPU (Tegra K1). A Comparison between SoCs and microprocessors is shown below (Table 1$)^{[20]}$.

\section{II.3.Enabled Optical Interconnects For Utility Computing And System Validation II.3.1.Photonic Networks for Hardware Accelerators:}

Hardware Accelerators normally needs high bandwidth, low latency, and energy efficient. The highperformance computing systems has critical performance is shifted from the microprocessors to the communications infrastructure. By uniquely exploiting the parallelism and capacity of wavelength division multiplexing (WDM), optical interconnects that able to address the bandwidth scalability challenges of future computing systems (Figure 5). The multi-casted network that uniquely exploits the parallelism of WDM to serve as an initial validation for architecture. Two FPGA boarded systems emulate the CPU and hardware accelerator nodes. Here FPGA transceivers implement and follows a phase-encoder header network protocol. The output of each port is individually controlled using a bitwise XNOR of port's control signal. Optical packets are send through the network and execute switch and multicasting of two receive nodes with most reduced error ${ }^{[14]}$.

\section{II.3.2.High-performance computing and memory interconnects using silicon photonics:}

The silicon photonics platform provides low cost, low energy and small optical interconnects resulting in integration between computational components and optical interconnects. Developers are further investigating FPGA-enabled silicon photonic interconnects for computational and processor-to-memory interconnects on work with Oracle Certified Master (OCM) architectures. FPGA-based optical network interface can execute primitives compatible with OCM data transactions. This repackaging of computing services is known as utility computing. Usage of different hardware accelerators like x86 CPUs, FPGAs, GPUs is the heterogonous computing within systems. High-bandwidth connectivity provided by WDM optical interconnects is an enabler for delocalized hardware accelerators in heterogeneous utility computing. Building a testbed in optically connected hardware accelerator emulators by FPGA-based system. In overall system design, the interconnection between the processor and memory is also taken into consideration. The memory system design used access many memory devices in parallel and transmit data over a high-speed, wide electronic bus and path-length-matched ${ }^{[6]}$. Optically-connected memory systems having high bandwidth capacity, energy efficient bit-rate transparency and time-of-flight latency nature. Optically-connected memory system provides an optical link between processing cores and main memory across an optical interconnection network. Effective silicon photonic modulators, filters and switches have been provide the device level for high-bandwidth and dynamic functionality but lacks unified and well-developed integration. By integrating control plane logic with device-level functionalities such as WDM, wavelength routing and broadband spatial switching are established in these systems (Figure 6) ${ }^{[7]}[21]$.

\section{II.3.3Optical network interface and protocol development:}

Maximal optical network utilization can be achieved by intelligently arbitrating wavelength routing and spatial circuit switching. Compatible with protocols specifically to operating in silicon photonic devices having programmable interface must implement all the required network protocols. Such closely integrated hardwaresoftware design is enabled by FPGA-based optical network interface (ONIC) logic. For established optical links through standard SerDes/electrical transceivers and data delivery mechanisms which is coupled with JTAG- and Ethernet-connected software co-processors, the ONIC employs logic to control wavelength stabilization and routing patterned data validation (PRBS and BERT) (Figure 7) ${ }^{[7]}$. 


\section{II.3.4Speeds up FPGA-in-the-loop verification:}

HDL Verifier used to speed up FPGA-in-the-loop (FIL) verification. Faster communication between the FPGA board and higher clock frequency stimulated by the FIL capabilities. This will increase the complexity of signal processing, control system algorithms and vision processing. For validate the design in the system context simulate hardware implementation on an FPGA board. HDL Verifier automates the setup and connection of MATLAB and Simulink test environments to designs running on FPGA development boards. The R2016b release allows engineers to specify a custom frequency for their FPGA system clock with clock rates up to five times faster than previously possible with FIL. This improves faster run-time. From MATLAB and Simulink is an easy way to validate hardware design within the algorithm development environment ${ }^{[8]}$.

\section{II.4. Low Power And High Data Rate Fpga:}

"Microsemi" FPGAs provides a non-volatile FPGA having $12.7 \mathrm{~GB} / \mathrm{s}$ transceiver and lower poor consumption less than $90 \mathrm{~mW}$ at $10 \mathrm{~GB} / \mathrm{s}$. It manufactured using a $28 \mathrm{~nm}$ silicon-oxide-nitride-oxide-silicon nonvolatile process on standard CMOS technology. By this they address cyber security threats and deep submicron single event upsets in configuration memory on SRAM-based FPGA. These transceivers cynical I/O gearing logic for DDR memory and LVDS. By cryptography research's produces differential power analysis protection technology, an integrated physical unclonable function and 56 kilobyte of secure embedded non-volatile memory, the built-in tamper detectors parts and counter measures.

\section{II.5. Fpga Board for Raspberry Pi:}

IceZero board is a "BML" creation that combines the best features of "IcoBoard", "BlackIce" and "IceHat". Its features fully open-source hardware and software design. The Lattice ICE40HX4K FPGA that supports Project IceStorm tool chain. Interfacing with Raspberry Pi 2x20 GPIO header for both power and interfacing bus. There is no need for JTAG programmer required, PROM directly programmed from Raspberry Pi. The external SRAM and extra-large SPI PROM supports soft CPU core designs for code execution and code storage respectively. It has an Industry standard PMOD expansion headers.

\section{IV.1.Altera Cyclone's Soc Flight Controller:}

\section{Recent Applications Of Fpga}

OcPoC (Octagonal Pilot on Chip) is the SoC having FPGA-based open-source flight control platform engineered to brings greatly enhanced I/O capabilities and processing power that is unparalleled by any other platform with enhanced GPS. OcPoC also expands its input and output capabilities to include fully programmable PWM, PPM and GPIO pins to integrate with different number of sensor (IMU). It having industry-leading more than $100 \mathrm{I} / \mathrm{O}$ for sensor integration, FGPA for sensor fusion, real time data processing and deep learning. Drone developers (3D modelling) can integrate various sensors and have the processing power to implement real-time processing of sensor data simultaneously. OcPoC confined other standardized connectors for peripherals such as CSI, GPS, camera link and SD card (Figure 8) ${ }^{[15]}$.

\section{IV.2.Ice40 ULTRAPLUS FPGA DEVICES:}

Lattice Semiconductor's iCE40 UltraPlus FPGA devices are claimed to be one of the most energyefficient parallel processing and programmable mobile heterogeneous computing (MHC) solutions. This device delivers eight times more memory as 1.1 Mbit RAM, twice the digital signal processors as 8x DSPs and improved I/Os over previous generations. It support voice recognition, gesture recognition, image recognition, haptics, graphics acceleration, signal aggregation or I3C bridging and more. It brings added intelligence to smartphones and IoT edge products such as drones, wearables, Human-Machine interfaces (HMIs), industrial automations, surveillance and home audio assisted devices. Its important feature is it is not needed to be always connected with cloud for interactions. This iCE40 applied in pedometer is shown below (Figure 9).

The MHC paradigm is a highly energy-efficient method for computing algorithms quickly and locally using dissimilar processors to offload power hungry application processors in battery-powered devices. Adding more DSPs offer the ability to compute higher-quality algorithms with noiseless. The flexible I/Os enable a more distributed heterogeneous processing architecture which provides flexibility to enable OEMs to quickly deliver key innovations. The device draws less than 100 micro watt in standby where these sensors able to operate all time ${ }^{[16]}$.

\section{Iv.3.Microsoft's Fpga Translates Wikipedia In Less Than A Tenth Of A Second:}

Microsoft's FPGA (Altera) system i.e. Altera translated every article on Wikipedia in less than a tenth of a second which holds 3 billion words. Here FPGA used for tuning or optimized specific task which improve its algorithm in hyper-scale applications. This flexible FPGAs accelerate servers in their massive datacenters. Microsoft is already using FPGA to power its search engine for upsurge search capabilities. Microsoft servers 
powered by optimized custom chips. This leads to artificial intelligence initiative for the Microsoft (Figure 10) [17]

\section{Iv.4.Risc-V Test and Verification Solutions:}

RISC-V is the instruction set architecture that becomes a standard open architecture for industry implementations under the governance. RISC-V is a compact ISA that is ideal for embedded applications including low-power platforms for the IoT. The main objective for the CPU is to implement a variety of performance enhancement such as pipelines, multiple instruction issue, out-or-order execution, branch prediction and caching. It defines the capabilities test program needs from the processor and memory. Its target environments as multicore, virtual memory and interrupts to set of allowed instructions and registers, execution start and end, test data input and test output data. RISCV-Torture generates random assembly tests ${ }^{[19]}$.

\section{Iv.5.Fpga Fabric Offered For Tsmc:}

A reconfigurable FPGA cores for implementation in TSMC's 40nm ultra-low power manufacturing process. They are moving its higher capacity EFLX-2.5K cores from TSMC's $28 \mathrm{~nm}$ CMOS process to the $16 \mathrm{~nm}$ FinFET processes. At $16 \mathrm{~nm}$ the fabric offers about $1 \mathrm{GHz}$ clock frequency under worst process as abnormal voltage and temperature conditions. In these applications there tends to be less arithmetic, more patternmatching and field-swapping gives more I/O capability and changed the size of the LUT to a 6-inputs from 4input design. The EFLX-100 IP cores can be arrayed in order to make larger arrays up to $5 \mathrm{X} 5$ with any combination of Logic and DSP IP cores inter-mixed. This covers from 120 LUTs up to 3000 LUTs. Networking, storage and security of data centers hardware protocols are reprogrammed as configurable-cloud data centers ${ }^{[18]}$.

\section{V.6. James Webb Space Telescope:}

The James Webb Space Telescope (JWST) view is 100 times larger compared to Hubble Telescope. The most important element on the telescope is the Near Infrared Spectrograph which having more than 250,000 micro-shutters. This micro-shutters have the ability to observe distant galaxies in space. The micro-shutters have micro-electro-mechanical system devices that shutter with light exposure, with the help of LabVIEW and Xilinx FPGA, the micro-shutters development of the electronic instruments that make up the JWST. The flexibility of FPGA allows designers to quickly adjust to changing image processing requirements and sensor interfaces.

\section{Iv.7. Uses Of Fpga:}

\section{IV.7.1.Uses Of FPGA In Green Technology:}

1) Solar Charge Systems

For optimal efficiency solar panel should be perpendicular to sunlight for strongest illumination. A sensor feedback FPGA controller is used to perform ADC. Then two motor controls to give 3D rotational Solar panel. Here two decoupled motors are used to give high efficiency and low power consumption.

2) Water treatment systems

Presently, Altera FPGA act as data acquisition system and control unit in treatment systems to give embedded operating system with digital filtering algorithm. Also FPGA powered Electromagnetic waves are used to break down lime scale deposits in the water and prevents oxidation on water piping systems.

3) Wind Energy

FPGA implemented Hardware-in-loop (HIL) emulator for wind turbine system developed for lab testing based on real time target generation. Based on the alternating input received certain control algorithms are initiated. FPGA-based sensor systems are also used to monitor the interaction between the turbines and the power grid, keeping power flowing smoothly into the grid.

4) Pollution control

Sensor-based FPGA technology to detect toxic gases. It is installed in vehicles to meet stringent vehicle air emissions standards. Using FPGA sensing unit is handled and ZIGBEE Module mounded with high frequency used to send quick intimations.

5) Water and air quality monitoring system

Measure water and air quality in polluted areas by FPGA-based systems. In a water monitoring system the number of variables are measured and these variables includes water's $\mathrm{pH}$ level. After collecting the values, it is remotely sends the data to a processing centre by FPGA-based sensor systems using ZIGBEE module. In similar way applied for air quality monitoring.

\section{IV.7.2.Uses for FPGA in Defense Systems:}

1) High-speed data processing systems

In Defense FPGA devices are used due to reduced risk, high performance, high capacity, high level of integration, highly safely and ANTI-Tamper technology. FPGA can be used to speed-up the data procession. Defense graded FPGAs has flexibility shows the endurance for heavy workload ${ }^{[3]}$. 
2) Electronic warfare systems

In electronic warfare the key drivers for continuous enhancement are Electronic-counter-counter-measures (ECCM).It has ability to rapidly analyze address multiple threads in short time frame. The electromagnetic spectrum is used to obstruct opponents and allow allies unhindered access to the EM spectrum. FPGA offer an ideal solution to these performance requirements in critical high-speed processing, to follow different electronic attack (EA) techniques ${ }^{[4]}$.

3) Tactical Communication Systems

Software defined ratios (SDR) used to re-configurability all transmissions data. FPGA are natural enablers of SDR applications.

4) Radar Systems

The FPGA are used to transform range-azimuth onto an $\mathrm{x}-\mathrm{y}$ coordinate plan in radar scan converter systems. There is a need for converting with high speed and accuracy of millions of data points.

\section{IV.7.3.Uses for FPGA in Original Equipment Manufactures:}

1) Machine to machine communication

In OEMs rely on machine vision applications in large scale factories use to build their products. These machines use vision-based systems to identify a familiar object and complete task/process was pre-programmed into its memory.

2) Image processing applications

Mobile robots are equipped with Image processing applications for detect any obstacles on its path ${ }^{[5]}$.

3) Sensors

Pressure sensors are used in modern traffic light systems, when the pressure system detects a vehicle's weight, it sends an alert FPGA-based control and sensing unit. Radiation sensors like Alpha, beta, gamma rays can be detected. An electric signal is received and then an actuator move or controlling a device ahead.

4) $\mathrm{M} 2 \mathrm{M}$

The FPGA system contains the sensors and products used to detect environment changers and processing Image processing.

\section{IV.7.4.Web-Based Services that use FPGA:}

1) FPGA computing hardware are used for data management services by Amazon's EC2 services, Cloudera, Google Compute Engine and Hortonworks. Microsoft Azure has recently stated that they will use FPGA as one of the main processing components behind their computing hardware.

2) Creating and host websites using in this FPGA platforms.

3) Software solution develop applications for mobile devices. Such Companies are Apache Cordova, Kendo UI and IONIC.

4) Some company provide e-commerce solutions for growing businesses. Such companies are, Magento, Prestashop and Shopify.

5) The companies provide the planning for enterprise resources. Such companies are CRM providers, SalesForce, HubSpot and Oracle ${ }^{[12]}$.

\section{Figures and Tables}

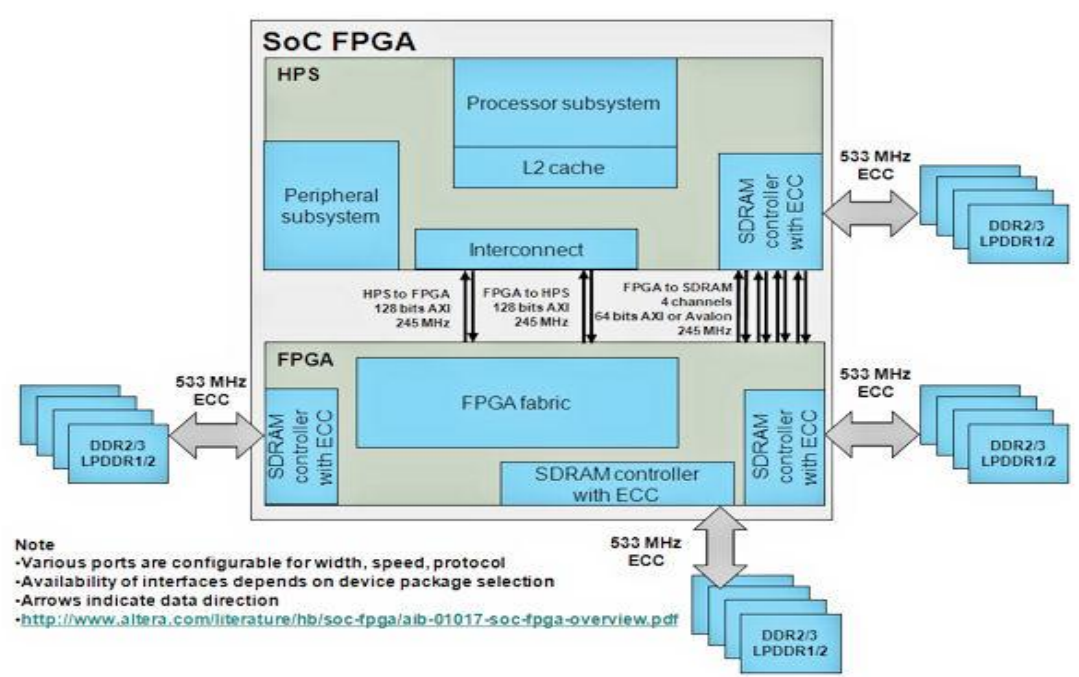

Figure 1: Architecture of FPGA. 


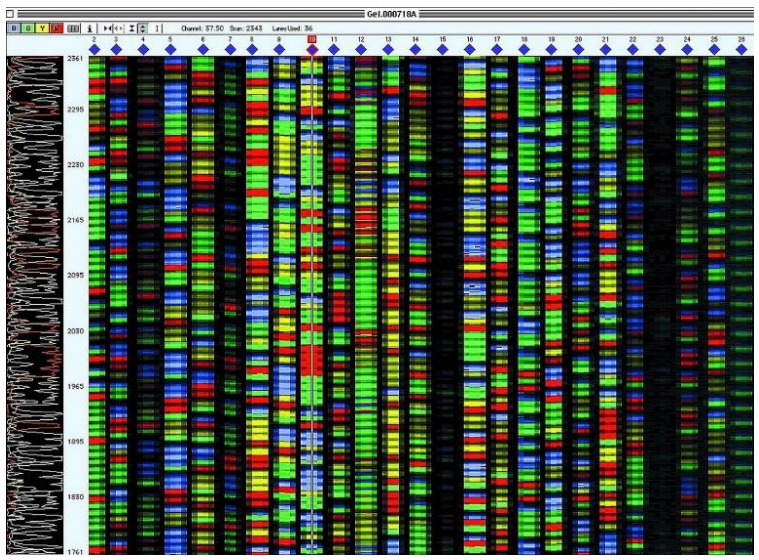

Figure 2: Gene Sequencing

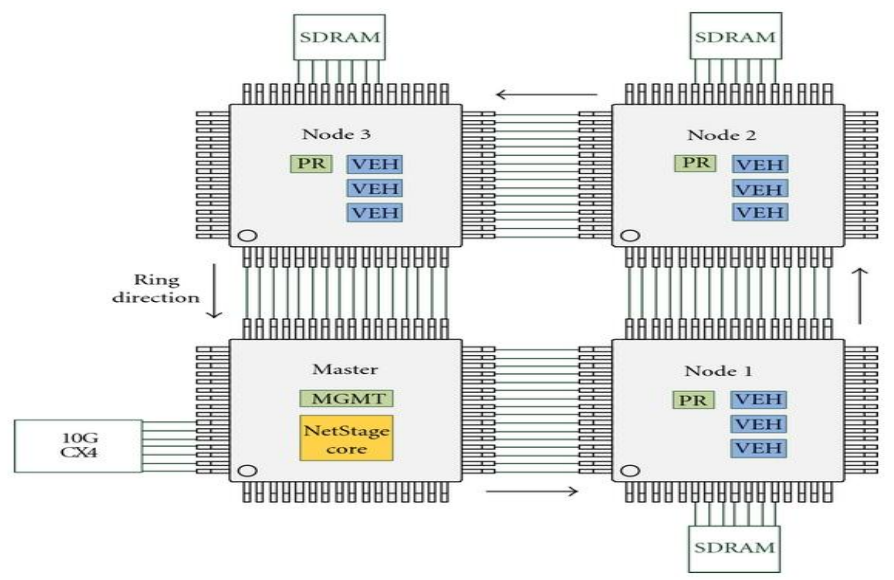

Figure 3: Multi-FPGA network in Ring Topology

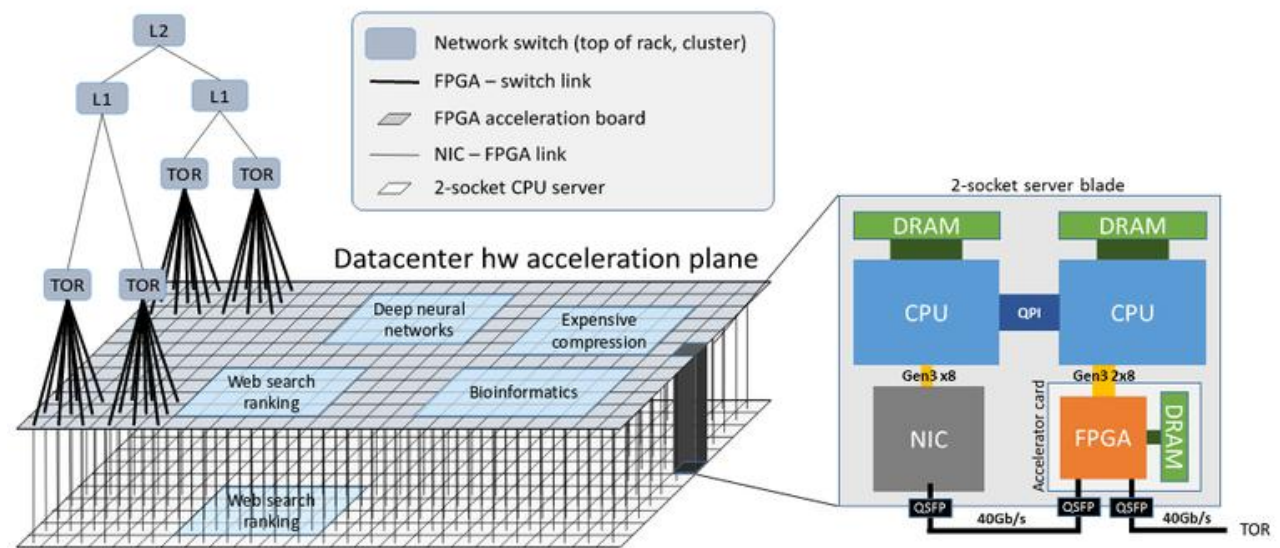

Traditional sw (CPU) server plane

(a)

(b)

Figure 10: Microsoft Azure cloud upgraded with FPGA

Table 1: Comparison table for Soc and MPs

\begin{tabular}{|l|l|l|l|}
\hline Target & Frequency & Performance & Energy efficiency \\
\hline Quad ARM A7 & $900 \mathrm{MHz}$ & $480 \mathrm{images} / \mathrm{s}$ & $380 \mathrm{images} / \mathrm{s} / \mathrm{W}$ \\
\hline Quad ARM A15 & $200 \mathrm{MHz}$ & $870 \mathrm{images} / \mathrm{s}$ & $350 / \mathrm{s} / \mathrm{W}$ \\
\hline Tegra K1 & $850 \mathrm{MHz}$ & $3,550 \mathrm{images} / \mathrm{s}$ & $600 \mathrm{images} / \mathrm{s} / \mathrm{W}$ \\
\hline Raspberry Pi 2B & $900 \mathrm{MHz}$ & $480 \mathrm{images} / \mathrm{s}$ & $380 \mathrm{images} / \mathrm{W}$ \\
\hline Odroid Xu3 & $2,000 \mathrm{MHz}$ & $870 \mathrm{images} / \mathrm{s}$ & $350 \mathrm{images} / \mathrm{W}$ \\
\hline PNeuroV2 (FPGA) & $100 \mathrm{MHz}$ & $7,000 \mathrm{images} / \mathrm{s}$ & $2,800 \mathrm{images} / \mathrm{s} / \mathrm{W}$ \\
\hline PNeuroV2 (ASIC) & $500 \mathrm{MHz}$ & $25,000 \mathrm{images} / \mathrm{s}$ & $125,000 \mathrm{images} / \mathrm{s} / \mathrm{W}$ \\
\hline
\end{tabular}




\section{Conclusion}

Since their introduction in 1985, field programmable gate arrays (FPGAs) have become significant role in the electronics industry. They have the potential for higher performance and compared with ASIC which offer lower non-recurrent engineering costs, reduced development time, easier debugging and reduced risk. Recently, FPGAs supports vertical migration and density migration. In this paper we see some recent developments using FPGA platforms and applications. FPGA concurred the major applications and hardware programmed devices. In our day-to-day life we come across many FPGA applicants with or without knowledge. Using HDL/Verilog it is easy to implement and customized our appliances.

\section{References}

[1] "Recent Trends in FPGA Architectures and Application” published by P.H.W. Leong, University of Sydney.

[2] "Recent Trends in FPGA Drive Adoption in Mil Apps" published by John Wranouics, Curtiss-Wright.

[3] "Today FPGA trends in aerospace and defence applications involve security, tools and specification" published by John Keller.

[4] "FPGA Trends Fuel Defence Design Organisation Rethink" published by J. Ryan Kenny, Altera.

[5] Special Issue "FPGA and SoC Devices Applied to new Trends in Image/ Video and Signal Processing Fields" by Dr. Ignacio Bravo-Munoz, Dr. Alferedo Gardel-Vincente, Prof. Dr. Jose L. Lazaro-Galileo.

[6] "Chips from Deep learning continue to leapfrog in capabalities and efficiency" by EETimes on 12/23/16

[7] Lightwave Reasearch Laboratory Columbia University in the city of New York published research on "FPGA- Enabled Optical Interconnects for Utility for Computing and System Validation"

[8] "MathsWorks Speed Up FPGA-in-the-Loop Verification" by Bussiness Wire.

[9] "Configuring the future for FPGAs in Genomics" published by Nicole Hemsoth.

[10] "FPGA Frontiers: New applications in reconfigurable computing" published by Timothy Prickett Morgan.

[11] “A Dynamically Reconfigured Network Platform for High-speed Malware Collection” by Sascha Muhlbhah

[12] "Uses of FPGA" Posted by Nadeesha Thewarapperuma.

[13] "Current trends in Hybrid FPGA/CPU devices" by Stephanie Rupprich.

[14] “A photonics Interconnection network for hardware accelerator enabled utility computing” by Cathy Chen, Howard Wang, Johnnie Chan and Keren Bergman, Columbia University.

[15] "SoC Flight Controller- OcPoC" by Aerotenna.

[16] "ICE40 UltraPlus FPsGA devices" by latticesemiconductor.

[17] "Microsoft's FPGA translates Wikipedia in less than a Tenth of a Second" article by Mark Hachman.

[18] "FPGA Fabric offered for TSMC" article by Peter Clarke.

[19] "RISC-V Verification" by asureISG

[20] "Google Designing AI Processors" article by Rick Meritt

[21] "Silicon Photonics Memory Interconnects for Many-core Architecture" by Ke Wen, Hang Guan from Colombia University with David Donofrio and John Shalf from Lawrece Berkeley National Laboratory. 